ESJ Social Sciences

\title{
Potencial económico del turismo médico veterinario en Ciudad Juárez
}

\author{
Jesús Alberto Urrutia de la Garza \\ Universidad Autónoma de Ciudad Juárez, \\ Departamento de Ciencias Administrativas, Mexico \\ Elsa Aranda Pastrana \\ Universidad Autónoma de Ciudad Juárez, \\ Departamento de Ciencias Sociales, Mexico
}

Doi:10.19044/esj.2021.v17n5p114

Submitted: 19 November 2020

Accepted: 22 January 2021

Published: 28 February 2021
Copyright 2021 Author(s)

Under Creative Commons BY-NC-ND

4.0 OPEN ACCESS

Cite As:

Urrutia de la Garza J.A. \& Pastrana E.A. (2021). Potencial económico del turismo médico veterinario en Ciudad Juárez. European Scientific Journal, ESJ, 17(5), 114.

https://doi.org/10.19044/esj.2021.v17n5p114

\section{Resumen}

México, es uno de los países líderes en turismo internacional receptivo. Dentro la variada oferta turística que tiene México, el ramo del turismo médico se encuentra entre los principales motivos de visitas extranjeras. Una de las actividades económicas de Ciudad Juárez, Chihuahua es la atención médica a residentes de El Paso, Texas y Las Cruces, Nuevo Mexico en los Estados Unidos y suburbios de las ciudades mencionadas. Debido al alto costo que representa este servicio médico en los Estados Unidos de América es la razónpor la que existe una gran cantidad de consultorios médicos, odontológicos, hospitales, laboratorios clínicos y servicios médicos para mascotas. El objetivo de este trabajo es hacer una aproximación para conocer el potencial económico que tiene el turismo médico veterinario en Ciudad Juárez. Esta investigación es de tipo cualitativo se fundamenta en trabajo de gabinete y de campo. El trabajo de campo consiste en la obtención de información sobre precios y servicios que proporcionaron clínicas veterinarias y en entrevistas realizadas en Ciudad Juárez. La ciudad tiene un importante potencial en el turismo veterinario por lo que es necesario fomentar acciones de innovación y emprendimiento parte de los interesados y de los gobiernos municipal y estatal para emular las mismas acciones o una política pública 
para aprovechar este mercado creciente.

Palabras clave: Turismo de médico, turismo veterinario, Región Binacional, Ciudad Juárez-El Paso

\title{
Economic Potential of Veterinary Medical Tourism in Ciudad Juarez
}

\author{
Jesús Alberto Urrutia de la Garza \\ Universidad Autónoma de Ciudad Juárez, \\ Departamento de Ciencias Administrativas, Mexico \\ Elsa Aranda Pastrana \\ Universidad Autónoma de Ciudad Juárez, \\ Departamento de Ciencias Sociales, Mexico
}

\begin{abstract}
Mexico is one of the leading countries in receptive international tourism. Within the varied tourist offer that Mexico has, the branch of medical tourism is among the main reasons for foreign visits. One of the economic activities of Ciudad Juarez, Chihuahua is medical care for residents of El Paso, Texas and Las Cruces, New Mexico in the United States and suburbs of the aforementioned cities. Due to the high cost that this medical service represents in the United States of America, it is the reason why there are a large number of medical and dental offices, hospitals, clinical laboratories and medical services for pets. The objective of this work is to make an approximation to know the economic potential of veterinary medical tourism in Ciudad Juarez. This research is qualitative and is based on documentary and field work. The field work consists of obtaining information on prices and services provided by veterinary clinics and in interviews carried out in Ciudad Juarez. The city has significant potential in veterinary tourism, so it is necessary to promote innovation and entrepreneurship actions by the stakeholders and the municipal and state governments to emulate the same actions or a public policy to take advantage of this growing market.
\end{abstract}

Keywords: Medical tourism, veterinary tourism, Binational Region, Ciudad Juarez-El Paso

\section{Introduction}

México, es uno de los países líderes en turismo internacional receptivo, su posicionamiento se basa en la diversidad de sus atractivos y en la variada oferta de destinos. Para el país, al igual que otros en desarrollo, "el turismo 
representa una alternativa de crecimiento económico y una oportunidad de evolución social" (Benseny, 2007, p. 30). Dentro la variada oferta turística que tiene México, el ramo del turismo médico (TM) se encuentra entre los principales motivos de visitas extranjeras que a su vez posicionan al país como uno de los destinos más visitados de América Latina en esta actividad. El TM se entiende como "el proceso mediante el cual una persona viaja fuera de su país de origen para realizarse algún procedimiento médico" (Ayala et al., 2010).

Se atribuye el desarrollo y aumento de la demanda del TM y servicios complementarios en el país a una especialización del personal que a lo largo de los años ha adquirido características y capacitación específicas. Lo que en la actualidad se interpreta como una potencial fuente de ingresos, empleo y nuevas posibilidades en el campo de la innovación, además de una mejora en la calidad de vida para los mismos desarrolladores de la actividad, "si se tiene en cuenta que es una experiencia que puede ser recreada continuamente con la gestión del conocimiento y la tecnología" (Benavides, 2015, p. 67).

Por otra parte, al hacer una alusión a las comunidades que desarrollan esta actividad, se hace mención a Ciudad Juárez, Chihuahua, México (CJZ) como una de las principales ciudades fronterizas mexicanas que se encuentran en la línea divisoria con los Estados Unidos de América (EUA). Las ciudades de CJZ y la de El Paso, Texas (ELP) y suburbios conforman una región binacional de más de dos millones de habitantes. Una característica importante de esta región es un intenso flujo de personas y de vehículos, en el 2015 se registró un flujo mensual de 1'594,147 vehículos y de 523, 441 personas (Plan Estratégico de Juárez, A.C, 2015). Las razones principales de estos cruces entre estas dos ciudades se deben a actividades comerciales, de negocios y personales. Una de las actividades económicas entre ambas ciudades, es la atención médica, debido al alto costo que representa ese servicio en EUA, derivado de esto, existe una gran cantidad de consultorios médicos, odontólogos, hospitales, laboratorios clínicos, entre otros, además de clínicas y hospitales veterinarios que en CJZ se encuentran principalmente apostadas a lo largo de las calles cercanas a los puentes internacionales del lado mexicano. El objetivo de este trabajo es hacer una aproximación para conocer el potencial económico que tiene el turismo médico veterinario en Ciudad Juárez

CJZ recibe visitantes que residen en las ciudades norteamericanas aledañas a la frontera en búsqueda de servicios médicos, sanitarios y de salud humana o de animales, por lo que se considera como una urbe con prácticas de turismo receptor, siendo una actividad económica que aporta al desarrollo de la comunidad dentro de una dinámica globalizadora como lo menciona Marysela Coromoto (2007, s.p.) que "Durante las últimas décadas, el turismo como actividad económica ha experimentado sorprendentes ritmos de 
crecimiento que superan incluso a sectores tradicionales en algunos lugares del mundo, por lo que dicha actividad adquiere mayor relevancia mundial."

\section{Metodología}

Esta investigación se realizó a finales del 2018 y principios del 2019, es una investigación de tipo cualitativo se fundamente en tres acciones: Una de trabajo de gabinete que sirve para una fundamentación conceptual en que muestra antecedentes y revisión de literatura referente al turismo de médico; la segunda y tercera acciones son trabajo de campo, una consiste en la obtención de información sobre precios y servicios que proporcionaron clínicas veterinarias; la otra acción fueron entrevistas a 16 miembros de la Asociación Mexicana de Médicos Veterinarios en Pequeñas Especies local, los cuales aceptaron participar y representan el $47 \%$ de los afiliados a la misma, cabe mencionar que contestaron de manera parcial a las preguntas por estar ocupados o las personas que contestaron no eran los dueños o encargados del consultorio o clínica, cabe mencionar que respondieron con cierta reserva por temor a llamar atención de las autoridades hacendarias porque no emiten facturas a los extranjeros.

\section{Desarrollo \\ Bases teóricas}

Los hallazgos encontrados por Martínez et. al. (2017) sobre el TM en ciudades fronterizas como CJZ, describen la singularidad de este tipo de destinos al carecer de atractivos turísticos variados, por lo que, no pueden estudiarse bajo las mismas teorías sobre segmentación del TM y las características del producto ofertado, ya que varían de manera considerable a las de otros destinos. En este tipo de ciudades, "la demanda obedece principalmente a la búsqueda de atención médica por su buena calidad, precio accesible y cercanía a los Estados Unidos como principal mercado" (Martínez et al., 2017, s. p.). El mismo artículo, analiza la relación de la segmentación del TM propuesta por Goodrich \& Goodrich (1987), quiénes combinan la salud con los ingresos, así como las variables de mayor importancia en un destino, siendo éstas las que a continuación se detallan en la Figura 1. 
Figura 1. Variables de importancia para un destino de Turismo Médico

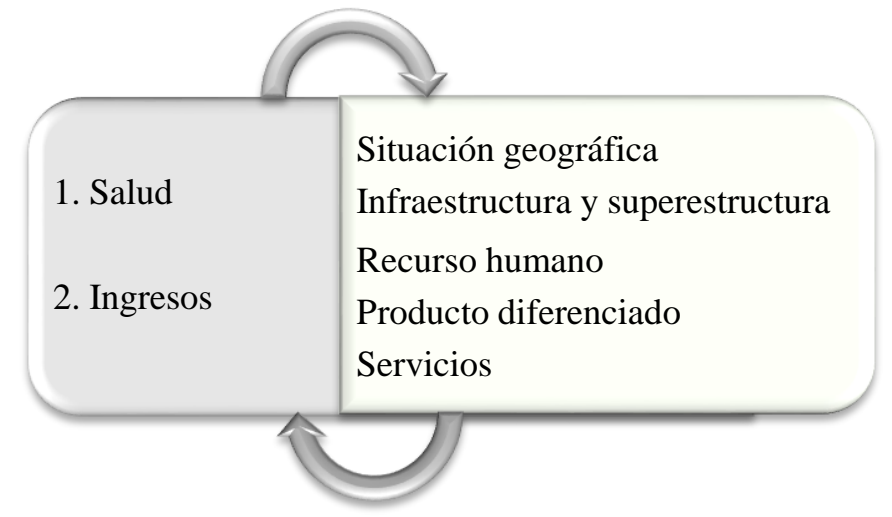

Fuente: Elaboración propia con base a Martínez et al. (2017).

La anterior Figura 1, muestra la importancia de la ubicación geográfica de esta ciudad fronteriza, radica en la cercanía con el mercado estadounidense le da una ventaja competitiva porque el tiempo traslado es mínimo y se disminuyen los riesgos que implica la realización de un viaje de cierto número de horas y menor costo para los pacientes (Martínez et al., 2017).

De la misma manera aborda otros elementos como "En cuanto a los aspectos psicográficos, la familiaridad con la gestión de cruce de frontera con la que cuenta el turismo de la diáspora es una ventaja que puede ser relacionada con la personalidad y gustos de los pacientes fronterizos, dado que este aspecto puede ser intimidante para pacientes internacionales" (Martínez et al., 2017, s.p.). También y en referencia al precio de los servicios, concluyen los mismos autores que "es uno de los factores más importantes de atracción de pacientes internacionales con problemas de salud, cuyos ingresos no son suficientes para cubrir sus gastos médicos en su país de residencia".

Por otra parte, (Heung, Kucukusta, \& Song, 2011) combinan los componentes de la salud y el turismo, así como los destinos que ofrecen opciones de atención sanitaria en paquete con visitas o paseos a sitios turísticos paradisíacos o reconocidos por su relevancia histórica. Mientras que (Alsharif el al., 2010) demuestran la importancia que tiene el costo de los servicios, la reputación del médico especialista y la acreditación del hospital para que el paciente tome una decisión.

Asimismo, bajo el mismo argumento de Gan y James (2011) que abordan la diferenciación del producto en el mercado del TM de los EUA afirmando que los facilitadores de turismo funcionan de manera similar a una industria de competencia monopolística y eligen diferenciar sus servicios de varias maneras, incluyendo la lista de países y hospitales que utilizan, el alcance de los tratamientos en los que se especializan, el grado de participación 
de los profesionales de la medicina en la empresa y los tipos de servicios auxiliares que ofertan.

También, Rerkrujipimol y Assenov (2011) mencionan como servicios diferenciadores, la oferta de transporte médico tanto aéreo como terrestre, apartamentos con servicios para los familiares acompañantes del paciente, cafeterías y restaurantes, servicio de inmigración en línea, traducción de diferentes idiomas, cuidado personal individual, atención personal de enfermería, servicios en línea para los arreglos médicos, centros de contacto, centro de paciente, la selección de hotel, reservas, servicios de excursiones de turismo, servicios de Internet y Wifi, entre otros.

Por último, Horton y Cole (2011) mencionan al segmento del mercado latino estadounidense como los principales compradores de estos servicios poniendo a consideración la determinante costo, la falta de seguro y conveniencia como impulso para buscar atención médica a lo largo de la frontera mexicana.

\section{Turismo de médico}

La búsqueda de tratamientos y procedimientos médicos fuera de las fronteras, por diferentes razones, ha creado una oferta internacional de servicios médicos, convirtiendo al TM en un sector de gran dinámica y crecimiento a nivel mundial (De la Puente, 2015). Esta práctica ha con llevado, dentro del contexto global, a la explotación de la diferenciación de precios entre el lugar de origen y el destino con una calidad, tecnología, protocolos y capacitación de personal similar que dan lugar a un sector alternativo (De la Puente, 2015)

Los países con economías emergentes como México son un atractivo para los habitantes pertenecientes a los países desarrollados debido a que en la actualidad los servicios médicos privados son de alta calidad y un precio más económico que en su país de origen. A pesar de que en otras épocas dichos países, recibían a personas de lugares con bajos y medianos ingresos en busca de atención especializada; actualmente, se han hecho famosos por su buena atención médica, tratamiento de enfermedades y rehabilitación, cirugía y otros procesos. (Alleyne, 1990; Vargas, 1993 y Lautier, 2008 citado en Arias et al., 2012; Ayala et al., 2010)

En la actualidad,según datos proporcionados por el Medical Tourism Index, México ocupa el lugar 29 a nivel mundial; el lugar número tres en industria del TM; el número 36 en la categoría de entorno turístico y la misma posición en la calidad de instalaciones y servicios.

\section{Turismo médico veterinario}

Diversos autores mencionan al turismo de salud desde dos vertientes muy claras, por motivos médicos o de bienestar, por lo que el concepto de TM 
es aquel que tiene como fin la realización de intervenciones médicas y médicoquirúrgicas favorables en costo menor y con servicios iguales, lo anterior se también se categoriza como bienestar (Arias et al., 2012).

Por tanto, dentro del TM que ofrece servicios especializados de salud para humanos, es posible clasificar los servicios médico-veterinarios o turismo médico veterinario (TMV), que si bien, no han tenido la misma demanda y popularidad haciéndolo un mercado poco explorado, lo cierto es que algunos estados en México y sobre todo fronteras han comenzado a enfocarse en este tipo de turistas, guiados principalmente por la derrama económica en el sector. Simplemente en el 2015, el mercado de mascotas se estimó en México en $\$ 1,778$ millones, y se prevé que alcanzará los $\$ 2,758$ millones para 2020, con una tasa compuesta anual de $9.18 \%$ durante el período de pronóstico (Diagnostics Technologies -Trends And Forecast, 2016)

El fenómeno del aumento en la preocupación por las mascotas se debe en gran parte, a que en la actualidad, los dueños de las mismas tienen más acceso a información y perciben la vida diferente a otros tiempos, desean brindarles existencias más largas, confortables y de alta calidad, lo que ha detonado una actividad económica que hace dos décadas no se percibían los productos y servicios que se pudiesen ofrecer como servicios veterinarios, de alimento, de ropa, de medicinas, así como estéticas, hoteles y servicios fúnebres (González, 2013).

El deseo de querer para las mascotas el mayor tiempo de vida y bienestar, es uno de los principales motivos que propician el TMV, siendo este factor el detonante de otras variables muy similares a las del turismo de médico para humanos, lo cual se basa en lo siguiente:

Figura2. Variables que inciden en el turismo médico veterinario

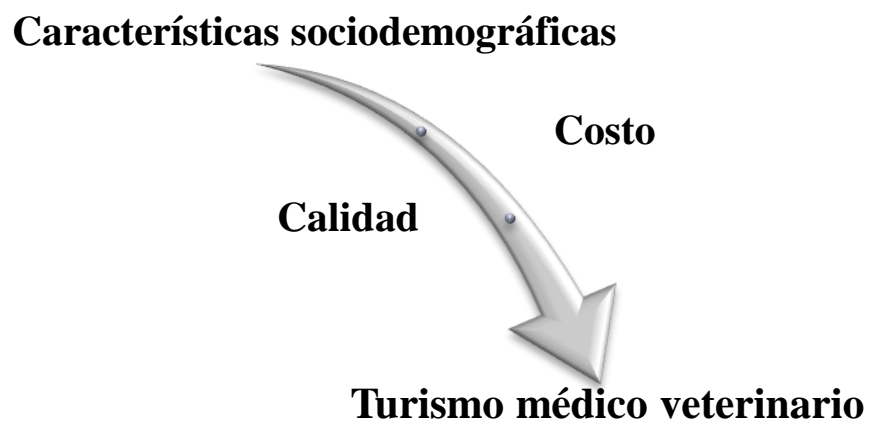

Fuente: Elaboración propia 2018

La Figura2 muestra como las características demográficas actuales que favorecen el fortalecimiento del TMV, ya que en los últimos años ha habido una creciente tendencia en tratar a los animales más allá que un "integrante de la familia", realmente lo hacen como si fueran "personas" (Franchise, s.f.).Por 
otra parte, los Baby boomers han reemplazado con mascotas a sus hijos como resultado del incremento de vida al nacer, de la estabilidad económica y que estos hijos hacen sus vidas de manera independiente. En casi todas las categorías de gastos, este disminuye una vez que una persona llega a los 55 años, pero el gasto de mascotas alcanza su punto máximo entre las edades de 55 y 64 años. Mientras que los Millennials por su parte, (personas nacidas entre 1985 y 2010) son probablemente la primera generación en crecer pensando en las mascotas más como humanos que como animales. Esta generación está encontrando su independencia, tienen ingresos disponibles, están comprando mascotas y las están malcriando (Franchise, s.f.).

En cuanto al costo y calidad, cabe resaltar que los principales turistas de servicios veterinarios en México provienen de Estados Unidos, realmente la atención de mascotas en ese país está sujeta a las mismas fuerzas del mercado que la atención de la salud humana: altos costos de mano de obra, medicamentos e instalaciones que generan gastos incosteables; por lo que al acudir a los veterinarios en México obtienen los mismos servicios por menos dinero, y estos ahorros pueden salvar vidas (The Baja Post, 2017).

Para las generaciones anteriores, cuando una mascota recibía un diagnóstico que amenazaba su vida, el siguiente paso era doloroso pero predestinado: la eutanasia. Hoy en día, cuando una mascota está muy enferma, comienza la toma de decisiones. Para un pequeño porcentaje afortunado de la población, gastar miles de dólares en un procedimiento veterinario avanzado y complejo no es un problema, pero para el resto, este estándar de cuidado va de la mano con la bancarrota (Entis, 2016). Entre los exámenes, las vacunas, las lesiones inesperadas y los medicamentos recetados, el costo de cuidar a una mascota pueden convertirse en una gran carga financiera para muchos propietarios (Woroch, 2017). La ciudad de ELP es una ciudad con una población hispana mayoritaria y los ingresos son relativamente bajos.

Los cinco diagnósticos más comunes cuestan en EUA alrededor de $\$ 1,000$ dólares para perros y gatos, a continuación, se detallan en la Tabla 1:

Tabla 1. Afecciones más comunes en mascotas

\begin{tabular}{|l|l|}
\hline Condición & Costo promedio (dólares) \\
\hline Ligamento/cartílago de la rodilla rasgado & $\$ 1,580.00$ \\
\hline Objeto extraño en el intestino & $\$ 1,970.00$ \\
\hline Objeto extraño en el estómago & $\$ 1,500.00$ \\
\hline Enfermedad del disco intervertebral & $\$ 3,280.00$ \\
\hline Torsión / hinchazón del estómago & $\$ 2,510.00$ \\
\hline
\end{tabular}

Fuente: AuWerter (2012).

Sin embargo, estos gastos pueden incrementarse dependiendo de las complicaciones. Por ejemplo, las dolencias estomacales pueden costar más de $\$ 6,000.00$ dólares para diagnosticar y tratar; los crecimientos y bultos más de 
$\$ 15,000.00$; las cirugías cardíacas pueden llegar a alcanzar los $\$ 20,000.00$ y las facturas mensuales de medicamentos pueden sumar más de $\$ 100.00$ dólares. Incluso las afecciones menos peligrosas pueden ser costosas, como infecciones de oído que pueden ser de hasta $\$ 250.00$ dólares por visita (Brophy, 2016).

\section{Turismo médico veterinario en la frontera de México}

Las ciudades en el país con mayor reconocimiento de ser proveedoras de servicios veterinarios a extranjeros son Tijuana en Baja California, Reynosa y Nuevo Laredo en Tamaulipas y posiblemente CJZ en Chihuahua. Desde hace algunos años han brindado este tipo de servicio a turistas provenientes de EUA, sin embargo, debido a que el mercado aun es relativamente nuevo, son acciones un tanto privadas, por lo que no se tiene registro de datos detallados referentes a la actividad del TMV.

En el 2008, el 60\% de los perros y gatos que recibían los veterinarios en estas ciudades provenían del extranjero (Buendía, 2014). El mismo autor menciona que debido a los altos índices de violencia, esta demanda disminuyó, sin embargo, en la actualidad se ha recuperado la confianza en los destinos y los extranjeros han regresado. Además, con el fin de recuperar los niveles de clientela, las diferentes autoridades y asociaciones han implementado medidas en sus ciudades, como la promoción en las páginas de Internet de los ayuntamientos y trámites para agilizar los cruces fronterizos de los clientes y sus mascotas.

En el caso de Tijuana, las consultas para mascotas proveniente de los EUA han incrementado en el 2017, hasta en un 30\%, los servicios veterinarios se han modernizado, y una de las razones por las que los extranjeros prefieren atenciones en esta ciudad, es que los costos son tres veces más baratos (Televisa Californias, 2018).

Según (Tapia citado en Rodríguez, 2018) alrededor del 20\% de las mascotas pacientes que reciben los veterinarios de Tijuana provienen de California, EUA, la razón, al igual que en el TM, en comparación resulta hasta $60 \%$ más económico tratar a las mascotas en centros veterinarios en la región, que del otro lado de la frontera. Sin embargo, también es por la calidad del servicio, pues a nivel mundial México está bien posicionado, inclusive hay veterinarios que ya cuentan con un pase médico para poder atender a pacientes del otro lado de la frontera.

Para el caso de Nuevo Laredo, se tienen en promedio entre cuatro a cinco consultas diarias de clientes extranjeros. La situación del dólar favorece a los clientes el país del norte ya que se ahorran al consultar a sus mascotas en Nuevo Laredo, entre un 20 y 30\% en servicios (Hernández, 2018). 


\section{Turismo médico veterinario en Ciudad Juárez}

Para el caso de CJZ el TMV se viene practicando desde varios años atrás, atendiendo mascotas provenientes principalmente de ELP. Sin embargo, es un mercado que no ha sido explotado en las mismas dimensiones de las ciudades antes mencionadas, ya que los veterinarios y clientes han tenido que lidiar en primer lugar con las limitantes en los cruces fronterizos y con la etiqueta de ser "la ciudad más violenta del mundo".

Hasta el 2007, previo a la crisis económica y de violencia que azotó a esta ciudad, la veterinaria Alma Rodríguez Giner, tesorera de la AMMVEPE, mencionó que los veterinarios cercanos a los puentes internacionales tenían dentro de su clientela aproximadamente un $80 \%$ de mascotas originarias de ELP. Sin embargo, con el repunte de la violencia en la ciudad, la clientela se desplomó, quedándose muy apenas con algunos clientes mexicanos. (Coronado, 2013)

Para el 2013, cuando esa etapa había sido superada los veterinarios continuaron con problemas, ya que no toda la clientela regresó, pero sí atendían en un 50\% a mascotas extranjeras; ahora sus principales limitantes eran el propio gobierno mexicano y de la oficina de Aduanas y Protección Fronteriza de Estados Unidos. Una razón que aun cuando los ahorros en servicios veterinarios representan más del doble para los turistas provenientes de EUA, la Aduana Mexicana solo permite cruzar dos perros de ELP a CJZ, cuando antes lo hacían hasta con seis (Coronado, 2013).

Aunado a lo anterior, las restricciones para el cruce de mascotas no se limitan solamente al número, sino que además requiere el presentar la cartilla de vacunación, cuando los propietarios cruzan principalmente a CJZ para recibir un servicio. Además, tanto propietarios como mascotas, tenían que esperar las largas filas en los puentes, tanto de ida como de regreso, por lo que, en algunas ocasiones, preferían mejor atenderlos en ELP al precio que costara (Coronado, 2013).

Las reuniones entre asociaciones de veterinarios y mesas de trabajo del sector económico en CJZ siguen mostrando un gran interés en el TMV, ya que no ha sido estudiado ni aprovechado en su totalidad y como ciudad destino de estos servicios comienza a quedarse rezagado; mientras que otras ciudades como en Nuevo Laredo, las reuniones entre especialistas están comenzando a gestionar el traslado de médicos del sur del país para realizar cirugías más complejas a las mascotas, como por ejemplo de corazón, en donde el propietario estaría dispuesto a pagar los gastos de viáticos y servicios con tal de salvar a sus mascotas (Nuevo Laredo Express, 2018).

Por tanto, CJZ con este importante potencial en el TMV, puede tomar como ejemplo las ciudades de Tijuana y Nuevo Laredo, para contactar y comenzar a emular las mismas acciones que tanto veterinarios como gobierno han emprendido para el aprovechamiento de este mercado creciente. 


\section{Resultados y discusión}

Los resultados aquí descritos, se distribuyen en dos secciones principalmente; I) percepción de los médicos veterinarios que trabajan con este mercados; II) comparativa sobre la diferencia de costos en los servicios que se ofertan en ambas ciudades. En primera instancia, se destacan los resultados de la aplicación las entrevistas para después presentar la comparativa en costos. Como se menciona en la metodología, el documento presenta una evaluación de las veterinarias en CJZ que se tomaron como muestra para el estudio.

Tabla 2. Comparativo de costos en CJZ - ELP

\begin{tabular}{|c|c|c|}
\hline Producto & Ciudad Juárez & El Paso \\
\hline $\begin{array}{l}\text { Paquete Perrito 1 } \\
\text { 5-en-1 (DAP + Parvo) } \\
\text { Desparasitante } \\
\text { Prueba fecal }\end{array}$ & $\begin{array}{l}\text { \$16.21 dólares } \\
\text { Cats\&Dogs }\end{array}$ & $\begin{array}{l}\text { \$60 dólares } \\
\text { Vip.petcare }\end{array}$ \\
\hline $\begin{array}{l}\text { Paquete Perrito } 2 \\
\text { 5-en-1 + Lepto } 4 \\
\text { Desparasitante } \\
\text { Bordetella nasal } \\
\end{array}$ & $\begin{array}{l}\text { \$18.64 dólares } \\
\text { Cats\&Dogs }\end{array}$ & \$69 dólares \\
\hline $\begin{array}{l}\text { Paquete Perrito } 3 \\
\text { 5-en-1 + Lepto } 4 \\
\text { Inyección de Bordetella } \\
\text { Desparasitante } \\
\end{array}$ & $\begin{array}{l}\text { \$18.64 dólares } \\
\text { Cats\&Dogs }\end{array}$ & \$69 dólares \\
\hline $\begin{array}{l}\text { Paquete de Perro } \\
\text { 5-en-1 + Lepto } 4 \\
\text { Bordetella } \\
\text { Prueba Fecal }\end{array}$ & $\begin{array}{l}\text { \$20.26 dólares } \\
\text { Cats\&Dogs }\end{array}$ & \$75 dólares \\
\hline $\begin{array}{l}\text { Paquete de Influenza } \\
\text { Canina } \\
\mathrm{H} 3 \mathrm{~N} 8+\mathrm{H} 3 \mathrm{~N} 2 \\
\end{array}$ & $\begin{array}{l}\text { \$10.81 dólares } \\
\text { Catalán }\end{array}$ & \$39 dólares Vip.petcare \\
\hline $\begin{array}{l}\text { Añadir } \\
\text { Vacuna de rabia para perro } \\
\text { válido para el paquete }\end{array}$ & $\begin{array}{l}\$ 8.10 \text { dólares } \\
\text { Catalán }\end{array}$ & \$15dólares Vip.petcare \\
\hline $\begin{array}{l}\text { Añadir prueba de gusano de } \\
\text { corazón / LYME } \\
\text { Válido para el paquete }\end{array}$ & $\begin{array}{l}\text { \$4.32 dólares } \\
\text { Veterinaria Fernández }\end{array}$ & +\$12dólares Vip.petcare \\
\hline $\begin{array}{l}\text { Paquete Gatito } 1 \\
3 \text {-en-1 } \\
\text { Desparasitante } \\
\text { Prueba fecal }\end{array}$ & $\begin{array}{l}\text { \$20.92 dólares } \\
\text { Veterinaria Fernández }\end{array}$ & $\begin{array}{l}\text { \$49 dólares } \\
\text { Vip.petcare }\end{array}$ \\
\hline $\begin{array}{l}\text { Paquete Gatito2 } \\
\text { 3-en-1 } \\
\text { Desparasitante } \\
\text { FeLV } \\
\text { (Añadir Prueba fecal por \$15) }\end{array}$ & $\begin{array}{l}\$ 21.50 \text { dólares } \\
\text { Veterinaria Ramírez }\end{array}$ & $\begin{array}{l}\text { \$56 dólares } \\
\text { Vip.petcare }\end{array}$ \\
\hline
\end{tabular}




\begin{tabular}{|c|c|c|}
\hline $\begin{array}{l}\text { Paquete Gatito3 } \\
\text { 3-en-1 } \\
\text { Desparasitante } \\
\text { Rabia De PureVax } \\
\text { FeLV } \\
\text { (Añadir Prueba fecal por \$ 15) }\end{array}$ & $\begin{array}{l}\text { \$18.64 dólares } \\
\text { Veterinaria Ramírez }\end{array}$ & $\begin{array}{l}\text { \$69dólares } \\
\text { Vip.petcare }\end{array}$ \\
\hline $\begin{array}{l}\text { Baño que incluye corte de } \\
\text { uñas, limpieza } \\
\text { limpieza dental, limpieza } \\
\text { glándulas anales, baño } \\
\text { antigarrapaticida y limpieza } \\
\text { nasal }\end{array}$ & $\begin{array}{l}\text { \$27.02 dólares } \\
\text { Veterinaria PeQ-es }\end{array}$ & $\begin{array}{l}\text { \$89 dólares } \\
\text { Pets Mart }\end{array}$ \\
\hline $\begin{array}{l}\text { Paquete Gato } \\
\text { 3-en-1 } \\
\text { Rabia De PureVax } \\
\text { Prueba Fecal } \\
\text { (Gatos que están expuestos al } \\
\text { aire libre según FeLV \$15) }\end{array}$ & $\begin{array}{l}\text { \$24.09 dólares } \\
\text { Hereford }\end{array}$ & $\begin{array}{l}\text { \$67 dólares } \\
\text { Vip.petcare }\end{array}$ \\
\hline $\begin{array}{l}\text { Paquete de operación } \\
\text { Fractura }\end{array}$ & & \\
\hline Rayos $\mathrm{x}+$ yeso & $\begin{array}{l}\text { \$70.27 dólares } \\
\text { Clínica Veterinaria González } \\
\text { Padilla }\end{array}$ & $\begin{array}{l}500 \text { a } 1200.00 \text { dólares } \\
\text { Pets Mart }\end{array}$ \\
\hline $\begin{array}{l}\text { Rayos } \mathrm{x}+\text { Clavos }+ \text { yeso } \\
\text { Rayos } \mathrm{x}+\text { Unión de } \\
\text { ligamentos }+ \text { Clavos }+ \text { Yeso }\end{array}$ & $\begin{array}{l}\text { \$108.10 dólares } \\
\text { Clínica Veterinaria González } \\
\text { Padilla. } \\
\$ 270.27 \text { dólares } \\
\text { Clínica Veterinaria González } \\
\text { Padilla. }\end{array}$ & \\
\hline $\begin{array}{l}\text { Paquete Rayos X } \\
3 \text { Tomas + tranquilizante }\end{array}$ & \$32.43 dólares & $\begin{array}{l}100 \text { a } 200 \\
\text { Eastwood Animal Clinic }\end{array}$ \\
\hline Operación de Estómago & \$189.18 dólares & $\begin{array}{l}\$ 1000 \text { a } 1500 \text { Eastwood } \\
\text { Animal Clinic }\end{array}$ \\
\hline
\end{tabular}

Fuente: Elaboración propia en base a trabajo de campo (2018-2019).

La anterior Tabla 2, muestra la gran diferencia que existe en los precios ofertados por las clínicas veterinarias de ambas ciudades, siendo CJZ más económica hasta en un $100 \%$ que la ciudad de ELP. Sin embargo, los resultados de la tabla reflejan mucho la relación del servicio con el precio es decir a intervenciones más complicadas aumenta el precio de una manera 
considerable en ambas ciudades siendo ELP mayor el porcentaje de aumento del precio. Se atribuye este fenómeno al factor riesgo y la probabilidad de lidiar con demandas ya que las leyes norteamericanas sí prevén la negligencia médica en los animales.

Por otra parte, se investigó en referencia a los principales servicios que proporcionan las clínicas resaltando que lo más recurrente son las vacunas, desparasitaciones, enfermedades respiratorias y gastrointestinales en las mascotas.

Asimismo se destaca en mayor número de incidencias en fracturas para mascotas que viven en CJZ atribuyéndole a las condiciones de la ciudad y la cultura de la misma.

Tabla 3. Percepción de los Médicos Veterinarios Zootecnistas sobre la operación de sus clínicas

\begin{tabular}{|c|c|}
\hline $\begin{array}{ll}\text { Entrevistado } 1 & \text { L.C }\end{array}$ & $\begin{array}{l}\text { "Atiendo bastantes personas que vienen de El Paso, sin } \\
\text { embargo, depende mucho la ubicación de mis clínicas } \\
\text { (tengo 4, únicamente } 2 \text { atiendo a turistas las otras son } \\
\text { para el mercado local). Me va muy bien gracias a Dios, } \\
\text { ya que he aumentado las ventas" }\end{array}$ \\
\hline Entrevistado 2 C.F & $\begin{array}{l}\text { "Por mi ubicación mi mercado es un } 80 \% \text { de El Paso, sin } \\
\text { embargo ya no es la misma clientela que en años } \\
\text { anteriores, una causa fue la inseguridad y otra la } \\
\text { competencia" }\end{array}$ \\
\hline Entrevistado 3 Dr. P. & $\begin{array}{l}\text { "Tengo una cartera de clientes bastante amplia, y por lo } \\
\text { general de muchos años atrás, si acaso atiendo un } 20 \% \\
\text { gente de El Paso. Creo que es por mi ubicación" }\end{array}$ \\
\hline Entrevistado 4 Dr. R. & $\begin{array}{l}\text { "Últimamente se bajaron muchos las ventas, considero } \\
\text { que en El Paso les están dando mejores condiciones para } \\
\text { que no se vengan para acá. Por mi ubicación me ha } \\
\text { mermado mucho la clientela. Queda pura gente vieja en } \\
\text { esta zona de la ciudad" }\end{array}$ \\
\hline Entrevistado 5 Dr. R1 & $\begin{array}{l}\text { "La ventaja competitiva que me ha mantenido con mis } \\
\text { clientes de Juárez y El Paso es el servicio las } 24 \text { horas, } \\
\text { atiendo } 50 \text { y } 50 \text { en mis dos clínicas }\end{array}$ \\
\hline Entrevistado 6 Dr. R2 & $\begin{array}{l}\text { "El mercado norteamericano se ha convertido en un } \\
\text { mercado muy peleado por los médicos veterinarios, } \\
\text { considero que no ha aumentado la afluencia de turistas a } \\
\text { las clínicas" }\end{array}$ \\
\hline Entrevistado 7 O.E.A & $\begin{array}{l}\text { "Tengo muy poco trabajo a pesar de estar en una buena } \\
\text { ubicación. No he aceptado bajar mis precios por lo cual } \\
\text { la gente se ha ido con clínicas más económicas" }\end{array}$ \\
\hline Entrevistado 8 & $\begin{array}{l}\text { "Desde mi punto de vista nos hace falta muchísima } \\
\text { organización para atender bien al mercado americano. } \\
\text { No lo vemos como turista, lo vemos como cualquier } \\
\text { cliente y no lo estamos explotando" }\end{array}$ \\
\hline
\end{tabular}

Fuente elaboración propia base trabajo de campo 2018. 
La Anterior Tabla 3. Muestra las principales percepciones de los médicos veterinarios dueños de las clínicas, los cuales demostraron un desconocimiento del término turismo médico en referencia a sus negocios, así mismo se encontraron opiniones encontradas en referencia a la rentabilidad de sus establecimientos ya que unos mencionan ser muy rentables y otros contestan lo opuesto totalmente. Por otra parte, se quejan de mucha competencia incluso culpando a las universidades de egresar demasiados profesionistas, lo que los exhibe como faltos de capacitación en áreas de mercadotecnia, administración y servicio al cliente, mismos factores que se ven reflejados en la falta de competitividad de sus negocios.

\section{Conclusiones}

Aunque, las veterinarias evaluadas, han establecido relación con sus clientes por periodos considerados lagos de tiempo. Las derivaciones apuntan a una mejor planeación en cuanto al desarrollo de los mismos, es decir llevar un mejor control del expediente médico de la mascota por parte de la empresa, así como mayor atención a factores de afiliación del turista dueño del animal con la empresa, como son la comodidad en la estadía, valor agregado en sus productos, llamadas pre y post consulta, personal bilingüe, invitación a cursos de manejo animal, entre otros y no únicamente el precio, además de una autoevaluación de las políticas de calidad en el servicio para trabajar aspectos de mejora continua.

De modo que la incorporación de estos factores en la gestión de las veterinarias y la medición en los mismos para hacerlos más controlables, se recomienda optimizar la competitividad de las clínicas mencionadas en CJZ, que a su vez se traduce en oportunidades para su aproximación al mercado tanto local, como internacional, (es decir no nada más la ciudad de ELP) debido a que actualmente se percibe una inexactitud en la incorporación de estrategias, lo que en el corto plazo perjudica la competitividad al empresario veterinario, posiblemente por falta de conocimiento del contexto sociocultural y económico-administrativo.

Este trabajo tiene un carácter exploratorio y es un primer estudio dentro de la parte económica en las veterinarias de CJZ con la intención de mejorar su competitividad. Su propósito es una primera aproximación para la gestión de la creación de una red formal con servicios complementarios para el turista que deja a su mascota en atención médica. Además, es necesario de examinar algunas de las razones básicas por las cuales dichas empresas en CJZ, no se consideran competitivas en comparación con empresas más grandes y con empresas norteamericanas. Sin embargo, se llega a una primera conclusión, el desarrollo y mejora de una red empresarial para este tipo de veterinarias se ve comprometido, si bien por una serie de factores, pero destaca la falta de 
organización por parte de los empresarios, además de infraestructura y cultura de afiliación por parte de los mismos.

Es necesaria la incorporación acciones más asertivas en la gestión de las veterinarias y la medición en los mismas para hacerlos más controlables, se recomienda optimizar la competitividad de las clínicas veterinarias. En definitiva, se requiere mayor profundidad en el tema y su promoción mediante nuevas investigaciones para revelar especificidades y necesidades de las veterinarias. Lo que ayudaría al debate, como otro motor económico para la ciudad, así como oportunidades de inclusión en la cadena de valor, temáticas que quedan por el momento fuera del alcance del presente documento.

\section{References:}

1. Arias, F., Caraballo, A., y Matos, R. (2012). Turismo de salud: conceptualización, historia, desarrollo y estado actual del mercado global. Clío América, Año 6, No. 11, pp.72-98. Consultado en https://dialnet.unirioja.es/servlet/articulo?codigo $=5114831$

2. Alsharif, M. J., Labonté, R., \& Lu, Z. (2010). Patients beyond borders: A study of medical tourists in four countries. Global Social Policy, 10(3), 315-335.

3. AuWerter, S. (2012). Reducing the Bite of Vet Care. Money, Vol. 41, No. 3, pp.27-29.

4. Ayala, C., Fadul, A., y Páez, F. (2010). Turismo en salud. Memorias del I Encuentro de Investigación Formativa 2010, Universidad Pontificia Bolivariana. Consultado de https://repository.upb.edu.co/bitstream/handle/20.500.11912/2731/Ar ticulo\%2020.pdf?sequence $=1 \&$ isAllowed $=y$

5. Benavides, G. (2015). Las políticas públicas del turismo receptivo colombiano. Suma de Negocios. Vol 6, No. 13, pp. 66-73.

6. Benseny, G. (2007). El turismo en México: Apreciaciones sobre el turismo en espacio litoral. Aportes y Transferencias. Vol. 2, Año 11, pp. 13-34.

7. Brophy, M. (1 de diciembre de 2016). Pet care costs can top human medical bills, new report reveals. Recuperado de https://www.cbsnews.com/news/pet-health-care-costs-can-tophuman-medical-bills-new-cat-and-dog-health-insurance-report/

8. Buendía, A. (30 de marzo de 2014). Crece turismo médico... de mascotas. El Norte. Recuperado de https://www.elnorte.com/aplicacioneslibre/articulo/default.aspx ?id=1 89772\&md5=75828a4c51e28d861e5aead1af80b4c9\&ta=0dfdbac117 $65226904 \mathrm{c} 16 \mathrm{cb} 9 \mathrm{ad} 1 \mathrm{~b} 2 \mathrm{efe} \& \mathrm{po}=4$

9. Coromoto, M. (2007). Análisis del turismo receptivo venezolano. Contaduría y administración, (222), pp. 115-135. 
10. Coronado, M. (29 de julio de 2013). Buscan abrir la frontera al turismo veterinario. Periódico El Diario. Recuperado de http://diario.mx/Local/2013-07-29_4d5d2499/buscan-abrir-lafrontera-al-turismo-veterinario/

11. De la Puente, M. (2015). Sector del turismo de salud: caso colombiano. Revista de Economía del Caribe. No. 16, pp. 129-161. Consultado en https://dialnet.unirioja.es/servlet/articulo?codigo $=6143212$

12. Diagnostics Technologies -Trends And Forecast. (2016). Mexico Veterinary Healthcare Market By Animal Types (Farm Animals, Companion Animals, Poultry) 2016-2021. Recuperado de https://www.researchandmarkets.com/research/tbsdn9/mexico_veteri nary

13. Entis, L. (8 de septiembre de 2016). When Fluffy's Surgery Costs More Than a Car. Recuperado de http://fortune.com/2016/09/08/pet-healthcare/

14. Franchise. (s.f.). Pet Care Industry Analysis 2018 - Cost \& Trends. Franchise Help. Recuperado de https://www.franchisehelp.com/industry-reports/pet-care-industryanalysis-2018-cost-trends/

15. Gan, L. L., \& James, F. (2011). Medical tourism facilitators: Patterns of service differentiation. Journal of Vacation Marketing, 17(3), 165183.

16. Goodrich, J., \& Goodrich, G. (1987). Health-care tourism - an exploratory study. Tourism Management, 217-222.

17. González, O. (16 de septiembre de 2013). Mascotas negocio millonario. El universal. Recuperado de http://archivo.eluniversal.com.mx/finanzascartera/2013/impreso/mascotas-105054.html

18. Heung, V., Kucukusta, D., \& Song, H. (2010). A Conceptual Model of Medical Tourism: Implications for Future Research.

19. Hernández, R. (19 de junio de 2018). Se mantiene turismo médico veterinario. Milenio Tamaulipas. Recuperado de https://www.pressreader.com/mexico/mileniotamaulipas/20180619/281758450008115

20. Horton, S., \& Cole, S. (2011a). Medical returns: Seeking health care in Mexico. Social Science \& Medicine 72 (2011) 1846e1852, 72, 1846-1852.

21. Martínez Almanza, M., Guía Julve, J., Morales Muñoz, S., \& Bribiescas Silva, F. (2017). Elementos del producto turismo médico en Ciudad Juárez, México. Revista Caribeña De Ciencias Sociales, (22547630). Retrieved from 
http://hdl.handle.net/20.500.11763/caribe1709turismo-medicomexico

22. Medical Tourism Index. Mexico Global Ranking. Consultado el 30 de agosto de 2018 en

https://www.microsofttranslator.com/bv.aspx?from=en\&to=es\&a=htt ps $\% 3 \mathrm{~A} \% 2 \mathrm{~F} \% 2 \mathrm{Fwww}$.medicaltourismindex.com $\% 2 \mathrm{Fdestination} \% 2 \mathrm{~F}$ mexico\% $2 \mathrm{~F}$

23. Nuevo Laredo Express. (16 de agosto de 2018). Atienden nuevo reto las veterinarias, la atención al turismo con sus mascotas. Recuperado de http://nuevolaredoexpress.com/atienden-nuevo-reto-lasveterinarias-la-atencion-al-turismo-con-sus-mascotas/.

24. Plan Estratégico de Juárez, A.C. Radiografía de la Economía Juarense. Ciudad Juárez: Plan Estratégico de Juárez, A.C. Recuperado de https://planjuarez.org/wpcontent/uploads/2018/12/economiaago2015.pdf

25. Rerkrujipimol, J., \& Assenov, I. (2011). Marketing Strategies for Promoting Medical Tourism in Thailand. Journal of Tourism, Hospitality \& Culinary Arts, 3(2), 95-105,. Retrieved from http://www.jthca.org/Download/pdf/V3 IS2/chap 8.pdf

26. Rodríguez, S. (19 de junio de 2018). Veterinarios de Tijuana son preferidos por las personas de California. Frontera.Info. Recuperado de

http://www.frontera.info/EdicionEnLinea/Notas/Noticias/19062018/1 350257-Veterinarios-de-Tijuana-son-preferidos-por-las-personas-deCalifornia.html

27. Televisa Californias. (21 de junio de 2018). Aumenta 30\% turismo médico veterinario en Tijuana. XEWT12. Recuperado de http://xewt12.com/aumenta-30-turismo-medico-veterinario-entijuana/

28. The Baja Post. (8 de julio de 2017). El turismo médico se extiende a los servicios de cuidado de mascotas. Recuperado de https://www.thebajapost.com/es/2017/07/08/medical-tourismextends-to-pet-care/

29. Woroch, A. (30 de agosto de 2017). Low-Cost Alternatives to Traditional Veterinary Care.Recuperado de https://money.usnews.com/money/personal-finance/saving-andbudgeting/articles/2017-08-30/low-cost-alternatives-to-traditionalveterinary-care. 